is ample scope for the employment of all the powers of observation and trained skill of a medical man, and only in his hands will they yield the best results and be comparatively free from the accompanying harmiul effects.

The following table summarizes the cases treated by high frequency alone.

\begin{tabular}{|c|c|c|c|c|c|c|c|c|}
\hline 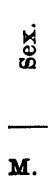 & 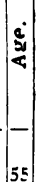 & \multicolumn{3}{|l|}{ Disease. } & 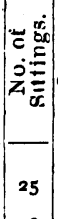 & \multicolumn{2}{|c|}{$\begin{array}{c}\text { Total } \\
\text { Duration } \\
\text { of Sictings. }\end{array}$} & Result. \\
\hline $\begin{array}{l}\mathbf{M} . \\
\mathbf{M} . \\
\mathbf{F} . \\
\mathbf{M} . \\
\mathbf{M} . \\
\mathbf{F} . \\
\mathbf{M} . \\
\mathbf{M} . \\
\mathbf{M} . \\
\mathbf{M} . \\
\mathbf{M} . \\
\mathbf{F} . \\
\mathbf{M} . \\
\mathbf{M} . \\
\mathbf{F} . \\
\mathbf{F} . \\
\mathbf{M} . \\
\mathbf{M} . \\
\mathbf{F} .\end{array}$ & \begin{tabular}{|r|}
55 \\
48 \\
44 \\
16 \\
23 \\
52 \\
29 \\
53 \\
27 \\
16 \\
43 \\
66 \\
25 \\
46 \\
28 \\
25 \\
19 \\
20 \\
30 \\
28 \\
6 \\
33
\end{tabular} & 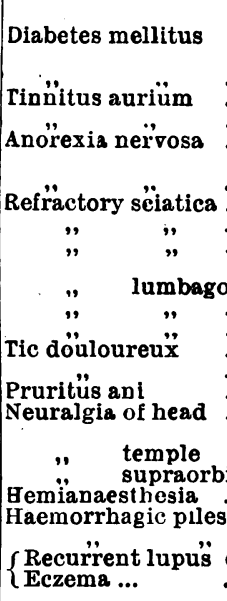 & $\begin{array}{l}\ldots \\
\ldots \\
\ldots \\
\ldots \\
\ldots \\
\ldots \\
\ldots \\
\ldots \\
\ldots \\
\ldots \\
\ldots \\
\ldots \\
\ldots \\
\text { bital } \\
\ldots \\
\text { erythem } \\
\ldots\end{array}$ & 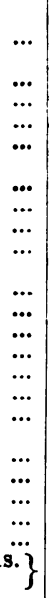 & \begin{tabular}{|r|}
25 \\
18 \\
10 \\
2 \\
5 \\
3 \\
9 \\
12 \\
2 \\
3 \\
5 \\
15 \\
5 \\
31 \\
10 \\
3 \\
5 \\
9 \\
13 \\
4 \\
2 \\
12
\end{tabular} & $\begin{array}{c}\text { Hr.s. } \\
4 \\
3 \\
5\end{array}$ & $\begin{array}{c}\text { Min. } \\
10 \\
0 \\
40 \\
20 \\
50 \\
30 \\
30 \\
15 \\
15 \\
20 \\
40 \\
20 \\
50 \\
10 \\
40 \\
15 \\
25 \\
45 \\
10 \\
40 \\
15 \\
10\end{array}$ & 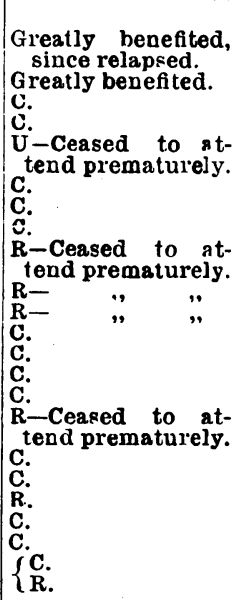 \\
\hline & & C-Cured. & R-Rel & lie & & & U- & eved. \\
\hline
\end{tabular}

\section{COLITIS POLYPOSA.*}

BY FRANK POPE, M.D., F.R.C.P., Senior Physician, Leicester In iirmary.

The following is a report of a case which presented a rare if not unique condition.

O. K., aged 21, was admitted to the Leicester Infirmary on January r6th, 1904 . She was wretchedly emaciated, had suffered severely from syphilis, had had an illegitimate child about nine months previously, followed by a septic condition of the passages and suppuration of Bartholin's glands.

\section{History of Illness.}

For six months she had had pain in the abdomen, coming on shortly after taking food, and continuing for about one hour and a-half, and during these attacks frequently vomited. She had alternately diarrhoea and constipation, and occasionally passed bright red blood in the stools. She had never vomited blood. Recently the bowels were very constipated, menstruation scanty. She had palpitation, headache, and dyspnoea on exertion.

Condition on Admission.

On examination there was some pain and tenderness in the epigastrium and right hypochondrium. There was retraction of the abdominal walls, but the diaphragm descended fairly well on inspiration, and there was little or no muscular rigidity. Nothing was felt on palpation. Temperature $98.6^{\circ}$, pulse 70 , very feeble. Mental condition weak, very emotional, but able to answer questions.

\section{After-History.}

The subsequent history may be summed up briefly by saying that she gradually sank, passed a good many small loosish motions, and at least one solid one. On January 20 th had a fit of unconsciousness (attributed to cerebral anaemia); she rallied somewhat from this, but died on the 23 rd, after twenty-four hours' further unconsciousness, apparently from asthenia.

\section{Necropsy.}

All thoracic organs and kidneys very pale, liver pale and fatty stomach and small intestine normal. Colon full, but not over-distended; its walls appeared externally to be somewhat thickened. On opening it the mucous membrane was found thickly covered with more or less pyramidal polypoid excrescences varying from the size of small shot to that of a field pea. There was no sign of ulceration. The majority of the growths were black at the apex. The growths were largest in the transverse portion of the colon; they extended into the upper part of the rectum, where they were less thickly distributed and more shot-like. There were no secondary deposits to be seen in any of the organs, but in front of the bodies of the lumbar vertebrae was a chain of slightly-enlarged lymphatic glands pinkish on section.

* Read at a meeting of the Midland Branch of the British Medical Association.
Mr. J. H. Targett reported

Pathological Report.

In the thickened part of the colon the mucous membrane is very rugose and villous, and the section shows that the cellular tissue of the mucosa and submucous coat is thickly infiltrated with small round cells. In consequence there is obstruction and dilatation of the gland follicles into small cysts.flled with thick secretion. The surface epithelium is detached, but there is no evidence of malignant disease. Much hypertrophy of the muscular coat exists, probably due to the constant irritation of the bowel. This condition of the large intestine may be described as "colitis polyposa," and it seems to be the result of a chronic inflammatory lesion.

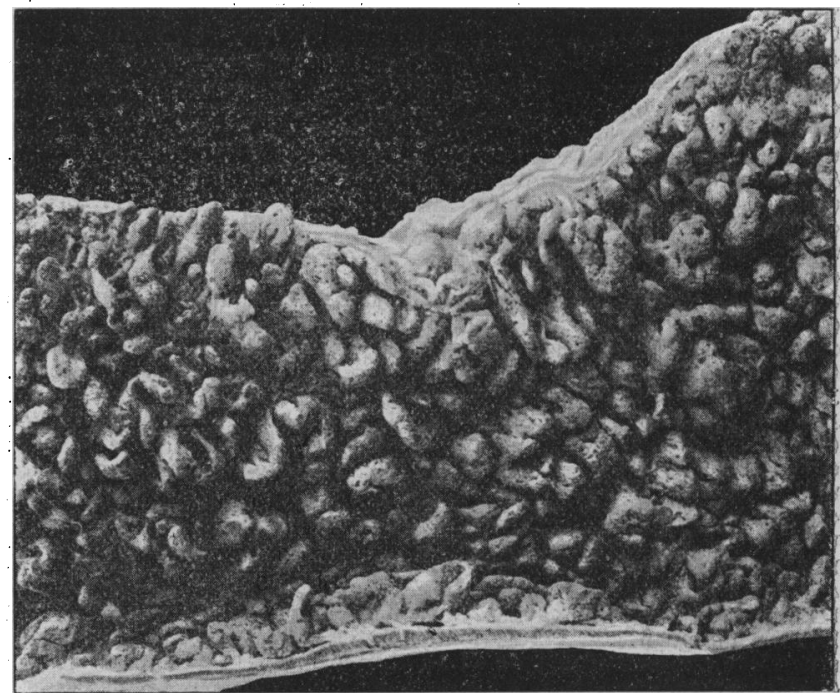

Fig. r.-Naked-eye appearances; from a photograph taken at the Royal College of Surgeons of England by Mr. H. George.

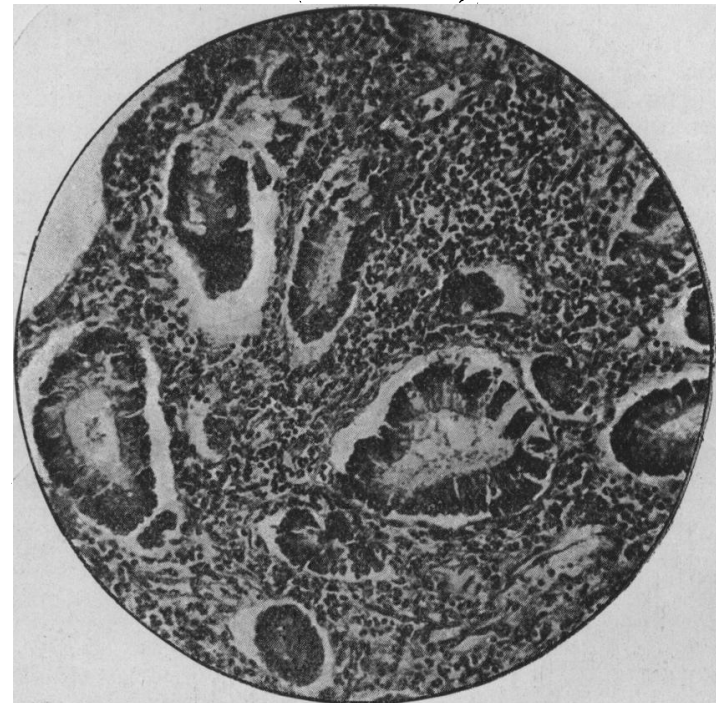

Fig. 2.-Microscopical section; from a photogreph taken by O. T. Elliott, Leicester.

I have not been able to find any reported cases resembling this. Norman Dalton reported one ${ }^{1}$ in which the tumours contained the elements of the mucous membrane in varying proportions, but there was no glandular proliferation. Cripps $^{2}$ reports a case from the Middlesex Hospital and figures the growth. It somewhat resembles the present one, but the polyps are rather more pedunculated. Cripps considers these to be adenoid in character. He also figures a mass of polypi of still more pedunculated form from King's College Museum. It is interesting to observe that the Middlesex Hospital case occurred in a man who had long suffered from a phagedaenic ulcer of the leg. He adds ${ }^{3}$ a most interesting account of similar growths in three members of one family, all young people; two of these had multiple adenomatous growths. The third, in addition to these, had developed a mass of adenoid cancer at the junction of the sigmoid flexure and rectum, from which he died. This is inter- 
esting in view of its suggestiveness as to the transformation of new growths from an innocent to a malignant form. Mr. Bond's case shown here to-day appears to be one of multiple adenomata.

Assuming that the polypoid condition in the present case was a direct result of septic conditions, we must recognize that it is possible to have a hypertrophic inflammation of the mucosa as well as the more common atrophic and destructive conditions met with in dysentery and the more usual forms of ulcerative colitis, and that as this condition of hypertrophy is not likely to have been of rapid growth, it is probable that the other septic processes in the patient were secondary to the colitis. It is likelv that some of the older cases regarded as adenomata were really of this nature, and three cases in one family rather suggest a common and infective origin.

I'bave little to say on the treatment. It must, I think, be mainly surgical, and colotomy and free washing out would be indicated were it not that the disease is not olten recognized until the patient has almost succumbed to its effects. It is very dêsirable that all cases of this nature should be reported so that their diagnosis may be facilitated. Mr. Targett informed me of disastrous effects in two cases which were the subject's of abdominal section in whom unsuspected ulcerative colitis existed. Attention should be especially directed to the early symptoms, and pain on going to stool and unexplained hatmorrhages extending over a long period should receive careful at:ention.

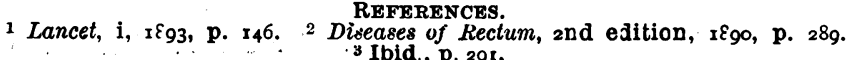

\section{A FATAL CASE OF CYANOSIS WITH ENLARGED SPLEEN.}

By THEODORE FISHER, M.D., M R C.P., Pathologist, Bristol Royal Infirmary; Physician to Out-patients,
Bristol Hospital for Sick Children.

AвоUT three years ago a case occurred in the post-mortem room of the Bristol Royal Infirmary to which, after the necropsy, the only heading that could be given to the notes was "Cyanosis, cause?" The cases mentioned by Dr. Osler in the BRITISB Medical Journal of January $16 \mathrm{th}, 1904$, and previously brought by him before the notice of the Association of American Phy sicians; have recalled this case of cyanosis to my memory.

A few notes of the case may be of interest.

A woman, aged 36 , was admitted under the care of Dr. H. Waldo, March ${ }_{2} 3^{\text {rd, }}$ rgor, with cyanosis which was said to bave existed for several years. It was stated at the time of the necropsy that her curious appearance repdered her an object of interest in the country district in which she had lived. Apparently she had, in spite of the cyanosis. enjoyed fairly good health, and, according to the clinical notes, suffered from nothing worse than attacks of bronchitis in the winter. She was married; but had nó children. About a month before admission she first noticed marked shortness of breath, and soon after some swelling of the legs appeared. At the time of admission the patient is described, in the clinical notes as " a heavy, obese woman, very blue in the 'face." There was some general oedema and the cyanosis affected the whole body. Some orthopnoea was present. examination of the lungs rales and rhonchi were widely heard. There was some dullness at the base of the right lung. The cardiac impulse could not be made out " nor the precordial dullness elicited on account of the obesity."' There were no murmurs. The pulse was regular, 96 . Nothing is recorded as having been noticed in the abdomen except the large amount of fat present. The urine was of specife gravity ro22 acid; a trace of albumen was present. Soon after admission 20 oz. Of The temperature remained normal until the day before death, when it ran

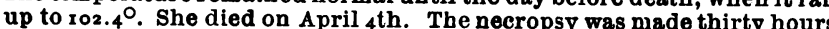
after death, and although the weather was not mild the amount of decomposition was far greater than is usually the case after that lapse of time. Blebs were scattered abundantly over the very stout body and the chest was marbled by staining in the situation of the subcutaneous veins. In the right pleural cavity were $25 \mathrm{oz}$. of fluid and a smalle quantity in the left pleural cavity. In the lungs there was nothing noteworthy. They were free from emphysema. The larynx, trachea, and bronchi presented nothing abnormal. The aorta was quite healthy, but the intima was much stained by the colouring matter of the blood. The pericardium was healthy but contained $6 \mathrm{oz}$. of fluid. The heart was dilated and measured 5 in. across the ventricles. The tricuspid and mitral orifices were, however, of normal size and the valves healthy. The cusps of the pulmonary and aortic valves were also quite healthy. The foramen ovale was closed, but it may be added that had it been patent, that would have been a feature of no special interest, since a valvular patency of the foramen ovale is found in about one heart in every four that is seen in the post-mortem room. The coronary arteries were also free from disease and the cardiac muscle, so far as could be seen, was quite heslthy, but unfortunately it was very much decomposed. The notes state that " although the patient is so very fat there is very little fat over the heart." By an unfortunate and most unusual oversight the weight of the heart has been omitted from the notes. Some hypertrophy was, however, present for which an explanation was not clear. The liver weighed $70 \mathrm{oz}$., was slightly congested, but except decomposition showed nothing noteworthy. The stomach was congested; the intestines showed nothing noteworthy. The adrenal bodies and pancreas, as well as the pelvic organs, were also healthy. The spleen was, however, enlarged and weighed $13 \mathrm{oz}$., but except for the enlargement showed nothing noteworthy. The kidneys were also much enlarged and weighed each $11 \frac{1}{2} \mathrm{oz}$. They are described as unfortunately being so decomposed as to be "useless for microscopical purposes." It is, however, added, "so far as could be seen there was nothing abnormal beyond their great size.'

The special features of this case, as seen in the necropsy room, were therefore, in addition to the cyanosis and obesity, an unusual amount of decomposition, some dilatation of the heart, an enlarged spleen, and greatly-enlarged kidneys. In the last detail the case seems to differ from the three cases recorded in Dr. Osler's original paper, inasmuch as in none of those cases was great enlargement of the kidneys observed. It is, perhaps, unfortunate that the extreme decomposition of the kidneys and of the cardiac muscle in this case led me to decide that microscopical examination would be valueless, but I think it may safely be said that, as has been mentioned above, it it highly improbable that anything noteworthy would have been discovercd that could not be seen with the naked eye.

In conclusion I have to thank Dr. Waldo for kind permission to record this case.

\section{MEDICAL, SURGICAL, OBSTETRICAL, THERA- pedtical, pathological, Etc.}

ACUTE INTESTINAL OBSTRUCTION CAUSED BY AN IMPACTED CONGERIES OF GALL STONES.

In the British Medical Journal of July 2nd, 1904, p. 19, Dr. E. C. Bridges communicates the case of a patient with "acute intestinal obstruction, caused by an impacted gall stone," in which Mr. D'Arcy Power operated. An experience of a somewhat similar case, with a similar result, vccurred to a patient of Dr. C. H. Caldicott, of Chobl am, Surrey, upon whom I operated.

History.-The patient, a man, aged 43, was siezed with sickness and vomiting and rather severe abdominal pains on June 21st, 1903, followed by a free action of the bowels; the pains continued until June 23 rd, when Dr. Caldicott was called in and found the patient suffering from intestinal obstruction. On June 24th Dr. F. M. Haig, of Woking, was called into consultation, and during the night of the 24th the patient was so collapsed that his life was despaired of. On June 25th I saw the patient with Dr. Caldicott, and found that he had rallied; the tongue was clean, the temperature $e_{r}$ and pulse normal, the abdomen somewhat distended, and the pain elicited by palpation slight and indefinite. Vomiting had occurred of a bilious character at long intervals. During my visit the patient passed some flatus and faeces, and, as he had rallied, operation was deferred. On the following day, June 26th, I visited the patient, but an hour previous to the time appointed for operation, the patient passed flatus freely and some small faecal lumps. He continued to pass flatus, the general condition was fairly good, and operation, after consultation, was again deferred.

Operation.-On June 28 th the improvement was not maintained, and laparotomy was decided upon. Assisted by Drs. Haig and Caldicott and Mr. P. L. Danitl, F.R.C.S., I opened the abdomen in the middle line below the umbilicus, and found the intestine distended as far as within some 18 in. of the ileo-caecal valve. A hard movable mass could be felt at the junction of the distended and collapsed portion of the intestine. The ileum was opened at this point; a rounded pear-shaped mass was removed and the intestine sutured, with but practically no disturbance of the abdominal contents. The calculus proved to be a conglomeration of small gall stones measuring $3 \frac{1}{2}$ in. in length and $I_{2}^{\frac{1}{2}}$ in. across at the blunt or lower end of the pear-shaped mass. There was no reason to anticipate a fatal issue, but, as in the case related by Dr. Bridges, the patient died in twelve hours after the operation.

REMARKS.-The gsmptomsin this case were interesting inas - 